\title{
Eva Teimel
}

Vorsitzende Fachausschuss und Gewerkschaft AHS

\section{Allen Menschen recht getan, ist eine Kunst, die niemand kann}

\section{Covid-19 aus der Sicht der Personalvertretung und Gewerkschaft}

\author{
DOI: https://doi.org/10.53349/sv.2021.i1.a60
}

\begin{abstract}
Covid-19 hat alle am Schulleben Beteiligten extrem gefordert und viel wurde auch den Lehrer*innen abverlangt - seitens des Ministeriums und der Bildungsdirektionen, der Eltern und der Öffentlichkeit. Für die Personalvertretung und Gewerkschaft war und ist das eine herausfordernde Zeit, im Spannungsfeld zwischen Systemrelevanz und drohender Überforderung des Berufsstandes der Lehrer*innen.
\end{abstract}

Covid-19, Standesvertretung, Gewerkschaft, Herausforderung

Drehen wir einmal kurz die Zeit in den Februar 2020 zurück: Covid-19 wütete in China (gefühlt ganz weit weg von uns) und schien uns nicht zu betreffen, dann in den März 2020: Die ersten Fälle traten in Österreich und sogar an einer niederösterreichischen Schule auf - damit war die Seuche schon ein gutes Stück näher an unsere bis dato heile Lebenswelt gerückt. Und gut erinnere ich mich an ein Treffen der Bildungsverantwortlichen in Niederösterreich mit der Standesvertretung der Schulen und Kindergärten Mitte März, als das erste Mal plötzlich von Lockdown, Schulschließungen und Homeschooling die Rede war - Wörter, die bis dato definitiv nicht in unserem aktiven Wortschatz vorgekommen waren.

Seien wir ehrlich: Wer hätte damals gedacht, was noch folgen würde? Viele dachten: Die drei Wochen bis Ostern im Homeschooling, okay, das wird jetzt nicht die Katastrophe werden, dann sind Osterferien und dann ist alles wieder vorbei. Ein naives Wunschdenken? In der Retrospektive betrachtet: ja. Die Osterferien gingen vorbei, die Pandemie nicht, ja sogar im Gegenteil: Sie wuchs sich aus und stellte alle (nicht nur die am Schulleben Beteiligten) vor völlig neue Herausforderungen und Probleme. 


\section{Die Rolle der Standesvertretung}

Als Personalvertreterin und Gewerkschafterin war man in dieser Situation extrem gefordert, denn letztendlich ging es darum, in vielen Dingen einen halbwegs gangbaren Mittelweg zu finden. Doch genau das war die größte Herausforderung: Zweifelsfrei gab es Kolleg*innen, welche die Pandemie damals nur für eine heftigere Grippewelle hielten und die sofortige Rückkehr vom Distance Learning in die Schulen forderten. Auf der anderen Seite standen diejenigen, die den Weg zurück in die Schule für undenkbar hielten - aufgrund eigener Vorerkrankungen, weil sie Risikopatient*innen in der eigenen Familie hatten oder sich persönlich fürchteten. Den einen gingen die Forderungen der Gewerkschaft zu weit, für die anderen forderte die Gewerkschaft zu wenig. Selten in der Geschichte der Standesvertretung waren die Meinungen und Ansichten der zu Vertretenden so weit gestreut (man denke an die Einigkeit der Kolleg*innenschaft beim Auftreten gegen die geplante Erhöhung der Lehrverpflichtung oder die Ablehnung des neuen Lehrerdienstrechtes etc.).

Als Fachausschuss- und Gewerkschaftsvorsitzende erreichten mich tagtäglich unzählige EMails und Anrufe, die oftmals in völlige diverse Richtungen gingen, von "Seid ihr wahnsinnig, wir müssen die Kinder sofort wieder zurück an die Schulen holen!“ bis „Seid ihr wahnsinnig, wir müssen die Kinder bis zu den Sommerferien daheim lassen!" Die Bandbreite der (oftmals wirklich berechtigten) Sorgen und (oftmals wirklich berechtigten) Begehrlichkeiten war enorm, die Diskrepanz zwischen den Extrempolen war groß und angesichts der völlig neuen und insgesamt unklaren Situation war man selber unsicher, was denn der richtige Weg wäre: Natürlich standen (und stehen) wir als Lehrer*innen aufgrund unseres systemrelevanten Berufs im Licht der Öffentlichkeit und sollten mithelfen, das „normale“ Leben halbwegs aufrechtzuerhalten; auf der anderen Seite standen die berechtigten Ängste der Kolleg*innen, die um ihre eigene und die Gesundheit ihrer Anverwandten fürchteten. Natürlich wollte das Gros der Lehrerschaft den Schüler*innen ermöglichen, möglichst rasch wieder an die Schulen und in die "Normalität" zurückzukommen, aber um welchen Preis?

Einen Umschwung brachte die politische Umsetzung einer Forderung von Gewerkschaft und Direktor*innen, die zunächst niemand für möglich gehalten hätte: die verpflichtenden Selbsttests der Kinder und Jugendlichen an den Schulen als Voraussetzung für den Schulbesuch sowie die FFP2-Masken-Pflicht für Schüler*innen der Oberstufe und für Lehrkräfte. Beide Maßnahmen steigerten das Sicherheitsgefühl der Kolleg*innen, die sich ebenfalls regelmäßig auch an der Schule testeten und FFP2-Masken trugen. Enorme Zusatzbelastungen wurden gestemmt, viel Mehrarbeit wurde geleistet - von laufenden Fortbildungen im digitalen Unterrichten bis zum oft sehr kurzfristigen Wechsel vom Distance Learning in den Schichtbetrieb, von zusätzlichen Aufsichtsdiensten über die Mitwirkung beim Testmanagement bis zum Abhalten von Ergänzungsunterricht bzw. notwendig gewordenen Förderkursen. Und kaum zu glauben: Was oftmals zu Beginn noch als unzumutbare Mehrbelastung wahrgenommen wurde, integrierten die Lehrer*innen bald in den Schulalltag. Es wurde ein selbstverständlicher Teil der Arbeit und stellte einen unverzichtbaren Beitrag zur bundesweiten Krisenbewälti- 
gung dar. Bleibt zu hoffen, dass dem Dienstgeber bewusst ist, wie viel aus Idealismus heraus geleistet wurde und dass diese Spitzenbelastung kein Dauerzustand bleiben darf.

\section{Und wie geht es weiter?}

Blicke ich auf die letzten Monate zurück, so wird mir diese Zeit in Hinblick auf meine Arbeit in der Standesvertretung wohl immer als eine besondere und besonders herausfordernde in Erinnerung bleiben: Täglich brachten mich unzählige E-Mails und Nachrichten auf vielen verschiedenen Kanälen und viele Telefongespräche in persönlichen Kontakt mit den von mir vertretenen Kolleg*innen (manchmal reichte der Akku des Handys nicht einmal einen Tag) - eine unverzichtbare Erfahrung. Auch wenn ich nicht immer eine Lösung parat hatte: Aktives Zuhören und der intensive persönliche Austausch haben so manche Sorge zerstreut und jedenfalls - auch bei unterschiedlicher Meinung - zu gegenseitigem Verständnis beigetragen.

Während ich diese Zeilen hier schreibe, sind die Corona-Zahlen massiv rückläufig, viele Menschen und ein Großteil der Kolleg*innen sind bereits geimpft, die Impfungen für Schüler*innen sind in greifbarer Nähe und es steht uns ein fast normaler Sommer ins Haus. Ähnliche Zeilen habe ich auch genau vor einem Jahr geschrieben - und dann traf uns Covid-19 im Herbst mit einer neuerlichen Heftigkeit und alles begann wieder von vorne. Mein unerschütterlicher Optimismus sagt mir: Im Herbst wird alles wieder gut. Und wenn nicht: Wir haben unsere Lektion gelernt: Wir Lehrer*innen lassen uns von Covid-19 nicht unterkriegen.

\section{Autorin}

\section{Eva Teimel, Mag.,}

unterrichtet seit 25 Jahren Latein und Geografie am BG/BRG Baden, Biondekgasse und ist Vorsitzende des Fachausschusses AHS und der AHS-Gewerkschaft NÖ. Kontakt: eva.teimel@bildung-noe.gv.at 